\title{
The Spatially Separated Polarization Sensitive FDA-MIMO Radar: A New Antenna Structure for Unambiguous Parameter Estimation
}

\author{
Binbin Li, Weixiong Bai, Qin Zhang, Guimei Zheng*, Mingliang Zhang, and Pengcheng Wan \\ Air and Missile Defense College, Air Force Engineering University, 710051 Xi'an, China
}

\begin{abstract}
Joint DOA-range-polarization estimation with a novel radar system, i.e., spatially separated polarization sensitive random frequency diverse array based on multiple-input multiple-output (SSPSRFDA-MIMO) radar, is discussed. The proposed array can obtain not only unambiguous range estimation but also polarization parameter estimation. Firstly, the signal model of SS-PSRFDA-MIMO radar is constructed. Secondly, dimension reduction multiple signal classification (DR-MUSIC) algorithm is extended to parameter estimation with the proposed array. Last, simulations demonstrate the proposed algorithm is effective to estimate parameter, and the performance of proposed array is better than that of polarization sensitive frequency diverse array based on MIMO radar. It is worth mentioning that the Cramér-Rao lower bound (CRLB) of range estimation with the proposed array is much lower than that of PSFDA-MIMO radar.
\end{abstract}

\section{Introduction}

In reference [1], we have discussed the direction of arrival (DOA), range and polarization estimation with polarization sensitive frequency diverse array based on multiple-input multiple-output (PSFDA-MIMO) radar. Although three algorithms are proposed to obtain accurate parameter estimation in reference [1], the problem of ambiguous range estimation is not considered. So, this paper attempts to solve the problem. To solve the problem of ambiguous range estimation with linear frequency diverse array (FDA), Wang et al. propose a random frequency diverse array in reference [2]. Motivated by that idea, this paper proposes a novel array, i.e., polarization sensitive random frequency diverse array based on MIMO radar (PSRFDA-MIMO) to solve the problem of ambiguous range estimation with PSFDA-MIMO radar. In order to reduce the mutual coupling, the spatially separated crossed-dipoles is chosen to be the array element of the proposed array. Therefore, the proposed array can be abbreviated as SS-PSRFDA-MIMO radar. Then a dimension reduction multiple signal classification (DR-MUSIC) algorithm is extended to parameter estimation with the proposed array. Compared with PSFDA-MIMO radar, the proposed array has following advantages: a) it can obtain unambiguous parameter estimation; b) the Cramér-Rao lower bound
(CRLB) of range estimation with the proposed array is much lower than that of PSFDA-MIMO.

\section{Signal model of SS-PSRFDA-MIMO radar}

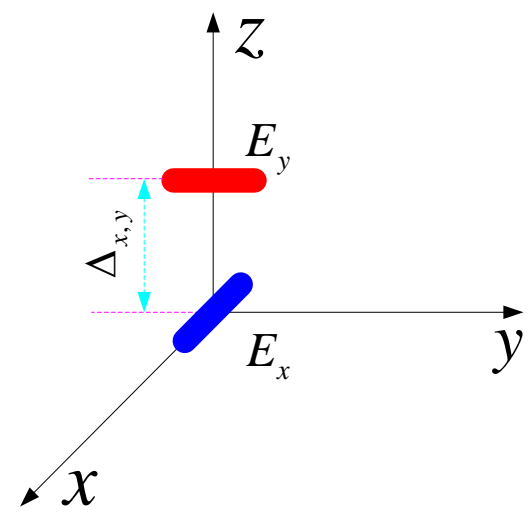

Fig. 1. Single vector sensor

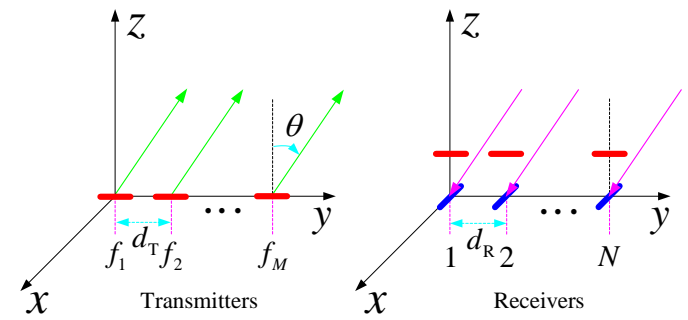

Fig. 2. Proposed SS-PSRFDA-MIMO radar.

\footnotetext{
* Corresponding author: zheng-gm@ 163.com
} 
Fig. 1 gives the single vector sensor (VS), which composes of spatially separated crossed-dipoles. The dipole $E x$ is oriented along the $x$-axis. The dipole $E y$ is oriented along the $y$-axis. $\Delta_{x y}$ is the space between $E x$ and Ey. The novel collocated MIMO radar is shown in Fig. 2. The transmitting array consists of $M$ dipoles separated by $d_{T}$. The receiving array consists of $N$ VSs separated by $d_{R}$. Direction of departure (DOD) and DOA are marked with $\theta$. Different from the conventional FDA, the carrier frequency of the transmitting array in the proposed array is random

$$
f_{m}=f_{0}+\omega_{m} \Delta f, \quad m=1,2, \cdots, M
$$

where $f_{0}$ denotes the center frequency, $\omega_{m}$ is a random variable obeying continuous uniform distribution, and $\Delta f$ denotes the frequency increment.

The response of the single VS can be expressed as [3]

$$
\begin{aligned}
& \boldsymbol{c}(\theta, \varsigma, \eta)=\underbrace{\left[\begin{array}{c}
1 \\
\mathrm{e}^{-\mathrm{j} 2 \pi \Delta_{x y} \cos \theta / \lambda}
\end{array}\right]}_{\underline{\underline{\Omega}} q(\theta)} \odot\{\underbrace{\left[\begin{array}{cc}
0 & -1 \\
\cos \theta & 0
\end{array}\right]}_{\underline{\underline{\Omega} \boldsymbol{\Omega}(\theta)}} \underbrace{\left[\begin{array}{c}
\sin \varsigma \mathrm{e}^{\mathrm{j} \eta} \\
\cos \varsigma
\end{array}\right]}_{\underline{\underline{\Omega}} \boldsymbol{g}(\zeta, \eta)}\} \\
& =\boldsymbol{q}(\theta) \odot[\boldsymbol{\Omega}(\theta) \boldsymbol{g}(\varsigma, \eta)]
\end{aligned}
$$

where $\varsigma$ and $\eta$ denote the auxiliary polarization angle and the polarization phase difference, respectively. $\odot$ denotes Hadamard product. The steering vector of the proposed array can be expressed as [1, 4-6]

$$
\begin{gathered}
\overline{\boldsymbol{a}}(\theta, r, \varsigma, \eta)=\boldsymbol{c}(\theta, \varsigma, \eta) \otimes \boldsymbol{a}(r, \theta) \otimes \boldsymbol{b}(\theta) \\
\boldsymbol{a}(r, \theta)=\boldsymbol{r}(r) \odot \boldsymbol{d}(\theta) \\
\boldsymbol{r}(r)=\left[\mathrm{e}^{-\mathrm{j} 4 \pi \omega_{1} \Delta f r / c}, \mathrm{e}^{-\mathrm{j} 4 \pi \omega_{2} \Delta f r / c}, \cdots, \mathrm{e}^{-\mathrm{j} 4 \pi \omega_{M} \Delta f r / c}\right]^{\mathrm{T}} \\
\boldsymbol{d}(\theta)=\left[1, \mathrm{e}^{\mathrm{j} 2 \pi d_{T} \sin \theta / \lambda}, \cdots, \mathrm{e}^{\mathrm{j} 2 \pi d_{T}(M-1) \sin \theta / \lambda}\right]^{\mathrm{T}} \\
\boldsymbol{b}(\theta)=\left[1, \mathrm{e}^{\mathrm{j} 2 \pi d_{R} \sin \theta / \lambda}, \cdots, \mathrm{e}^{\mathrm{j} 2 \pi d_{R}(N-1) \sin \theta / \lambda}\right]^{\mathrm{T}}
\end{gathered}
$$

where $\otimes$ denotes Kronecker product, superscript $(\cdot)^{\mathrm{T}}$ represents transpose of a matrix or vector. $\boldsymbol{a}(r, \theta)$ and $\boldsymbol{b}(\theta)$ represent transmitting and receiving steering vector, respectively. Thus the received data can be expressed as

$$
z(t)=\sum_{k=1}^{K} \overline{\boldsymbol{a}}\left(\theta_{k}, r_{k}, \varsigma_{k}, \eta_{k}\right) s_{k}(t)+\boldsymbol{n}(t)
$$

where $K$ denotes the number of targets. The echo signal $s_{k}(t)$ is assumed to be a zero-mean, complex Gaussian, random process. $\boldsymbol{n}(t)$ denotes a $2 M N \times 1$ complex Gaussian white noise vector with zero mean and covariance matrix $\sigma^{2} \boldsymbol{I}_{2 M N} \cdot \sigma^{2}$ denotes the noise variance. $\boldsymbol{I}_{2 M N}$ represents a $2 M N \times 2 M N$ identity matrix. Now, our work is to obtain the estimation of four parameters $\left\{\theta_{k}, r_{k}, \varsigma_{k}, \eta_{k}, k=1, \cdots, K\right\}$ according to the signal model of (8).

\section{Proposed dimension reduction (DR) MUSIC algorithm}

Obviously, there are four parameters to be estimated in the steering vector of (3). Generally speaking, the four parameters can be estimated using multiple signal classification (MUSIC) algorithm through fourdimensional searching

$$
f_{4 \mathrm{D} \text {-MUSIC }}=\frac{1}{\overline{\boldsymbol{a}}(\theta, r, \varsigma, \eta)^{\mathrm{H}} \boldsymbol{E}_{N} \boldsymbol{E}_{N}^{\mathrm{H}} \overline{\boldsymbol{a}}(\theta, r, \varsigma, \eta)}
$$

where $\boldsymbol{E}_{N}$ is noise subspace, which can be computed according to the received data of (8). However, the fourdimensional searching will result in heavy calculation. So, we will propose a dimension reduction MUSIC algorithm (DR-MUSIC) with two-dimensional searching. The detail process is elaborated as follows.

Utilizing the matrix formula $(A B) \otimes(C D)=(A \otimes B)(C \otimes D)$, the steering vector can be converted into

$$
\begin{aligned}
\overline{\boldsymbol{a}}(\theta, r, \varsigma, \eta) & =\boldsymbol{c}(\theta, \varsigma, \eta) \otimes \boldsymbol{a}(r, \theta) \otimes \boldsymbol{b}(\theta) \\
& =\{\boldsymbol{q}(\theta) \odot[\boldsymbol{\Omega}(\theta) \boldsymbol{g}(\varsigma, \eta)]\} \otimes \boldsymbol{a}(r, \theta) \otimes \boldsymbol{b}(\theta) \\
& =[\overline{\boldsymbol{\Omega}}(\theta) \boldsymbol{g}(\varsigma, \eta)] \otimes\left\{[\boldsymbol{a}(r, \theta) \otimes \boldsymbol{b}(\theta)] \boldsymbol{I}_{1 \times 1}\right\} \\
& =\{\overline{\boldsymbol{\Omega}}(\theta) \otimes[\boldsymbol{a}(r, \theta) \otimes \boldsymbol{b}(\theta)]\}\left[\boldsymbol{g}(\varsigma, \eta) \otimes \boldsymbol{I}_{1 \times 1}\right] \\
& =\underbrace{\overline{\boldsymbol{\Omega}}(\theta) \otimes[\boldsymbol{a}(r, \theta) \otimes \boldsymbol{b}(\theta)]\}}_{\underline{\triangleq}(r, \theta)} \boldsymbol{g}(\varsigma, \eta)
\end{aligned}
$$

where $\boldsymbol{I}_{1 \times 1}$ denotes a $1 \times 1$ identity matrix. Each column of $\overline{\boldsymbol{\Omega}}(\theta) \in \mathbb{C}^{2 \times 2}$ is equal to the Hadamard product of each column of $\boldsymbol{\Omega}(\theta)$ and $\boldsymbol{q}(\theta)$, i.e., $\overline{\boldsymbol{\Omega}}=[\boldsymbol{\Omega}(:, 1) \odot \boldsymbol{q}, \boldsymbol{\Omega}(:, 2) \odot \boldsymbol{q}]$. As we know, the problem of parameter estimation is actually that of maximizing Equation (9). Then it can also be transformed into a problem of minimizing the following cost function

$$
V=\overline{\boldsymbol{a}}(\theta, r, \varsigma, \eta)^{\mathrm{H}} \boldsymbol{E}_{N} \boldsymbol{E}_{N}^{\mathrm{H}} \overline{\boldsymbol{a}}(\theta, r, \varsigma, \eta)
$$

Due to the identical equation, i.e., $\boldsymbol{g}(\varsigma, \eta)^{\mathrm{H}} \boldsymbol{g}(\varsigma, \eta)=1$, Equation (11) can be rewritten as

$$
V=\frac{\boldsymbol{g}(\varsigma, \eta)^{\mathrm{H}} \boldsymbol{\psi}(r, \theta)^{\mathrm{H}} \boldsymbol{E}_{N} \boldsymbol{E}_{N}^{\mathrm{H}} \boldsymbol{\psi}(r, \theta) \boldsymbol{g}(\varsigma, \eta)}{\boldsymbol{g}(\varsigma, \eta)^{\mathrm{H}} \boldsymbol{g}(\varsigma, \eta)}
$$

Referring to the Rayleigh-Ritz theory [7], the problem of minimizing $V$ can be converted into that of solving the minimum eigenvalue (marked with $\alpha_{\text {min }}$ ) of the matrix $\boldsymbol{\psi}(r, \theta)^{\mathrm{H}} \boldsymbol{E}_{N} \boldsymbol{E}_{N}^{\mathrm{H}} \boldsymbol{\psi}(r, \theta)$. Thus DOA and range estimation can be obtained as follows through twodimensional searching

$$
f_{\text {2D-MUSIC }}=\arg \max _{\theta, r}\left[1 / \alpha_{\text {min }}(\theta, r)\right]
$$

The estimated polarization vector (marked with $\hat{\boldsymbol{g}}(\varsigma, \eta)$ ) is the eigenvector corresponding to the minimum eigenvalue. Then the polarization parameter can be computed as follows

$$
\begin{gathered}
\hat{\boldsymbol{\varsigma}}=\arctan \left|\frac{\hat{\boldsymbol{g}}(\varsigma, \eta)_{1}}{\hat{\boldsymbol{g}}(\varsigma, \eta)_{2}}\right| \\
\hat{\eta}=\angle \frac{\hat{\boldsymbol{g}}(\varsigma, \eta)_{1}}{\hat{\boldsymbol{g}}(\varsigma, \eta)_{2}}
\end{gathered}
$$




\section{Simulations}

To demonstrate the perfect performance of the proposed array, PSDFDA-MIMO radar is introduced for comparison. Assume that there are 10 array elements separated by half a wavelength in the transmitting and receiving array. The space of crossed-dipoles of single vector sensor is also set to be half a wavelength. Two non-coherent narrowband signal sources are considered:

$$
\begin{aligned}
& \left(\theta_{1}, r_{1}, \varsigma_{1}, \eta_{1}\right)=\left(10^{\circ}, 20 \mathrm{~km}, 45^{\circ}, 45^{\circ}\right) \\
& \left(\theta_{2}, r_{2}, \varsigma_{2}, \eta_{2}\right)=\left(20^{\circ}, 30 \mathrm{~km}, 60^{\circ}, 30^{\circ}\right) .
\end{aligned}
$$

Suppose that the probability density function (PDF) of $\omega_{m}$ is $1 / 2$. The center frequency is set to be $3 \times 10^{9} \mathrm{HZ}$. The frequency increment is set to be $10^{6} \mathrm{HZ}$. signal-tonoise-ratio (SNR) is set to be $20 \mathrm{~dB}$. The number of snapshots is set to be 1000 . Fig. 3 (a) and (b) show the spatial spectrum of proposed array and PSFDA-MIMO array based on DR-MUSIC algorithm, respectively. As shown in Fig. 3, the proposed array can give unambiguous parameter estimation, but the range estimation with PSFDA-MIMO array is ambiguous.

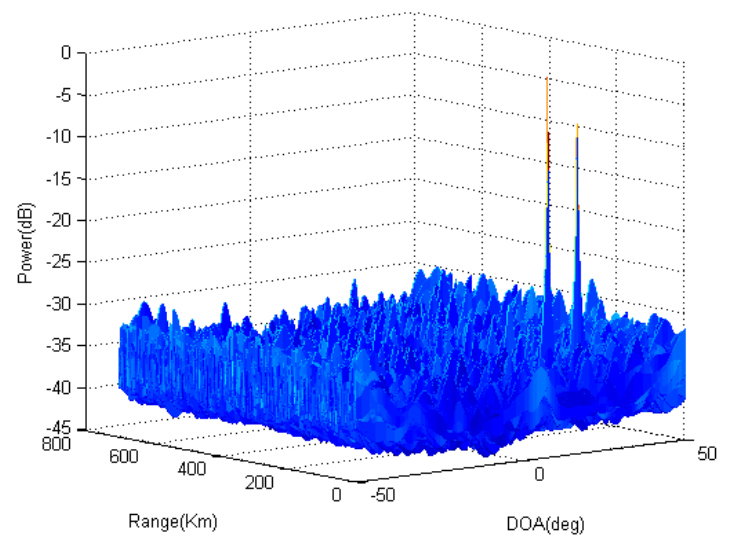

(a) Spatial spectrum based on proposed array

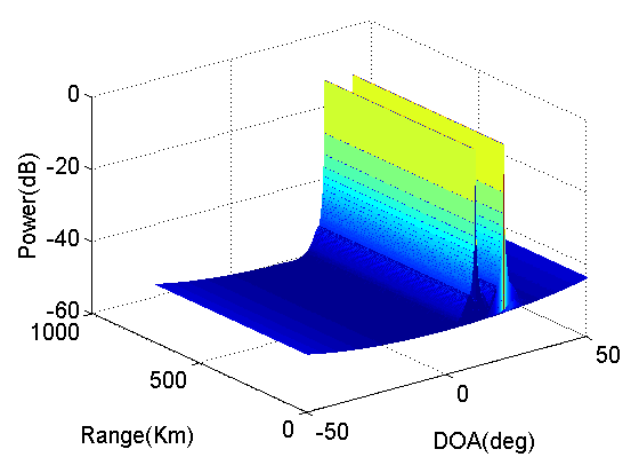

(b) Spatial spectrum based on PSFDA-MIMO radar

Fig. 3. Spatial spectrum based on two kinds of arrays

Next we will compare the CRLB based on proposed array with that based on PSFDA-MIMO radar to demonstrate the perfect performance of the proposed array. CRLB based on PSFDA-MIMO radar [1] has been derived. So, CRLB based on proposed array can be derived similarly. But we should substitute the joint steering vector of this paper into that of reference [1]. The number of snapshots and Monte Carlo experiment are set to be 1000 and 100 , respectively. SNR varies from $-10 \mathrm{~dB}$ to $10 \mathrm{~dB}$ with $2 \mathrm{~dB}$ interval. Fig. 4 shows how the root mean square error (RMSE) of parameters estimation varies against SNR. From the results in Fig. 4, we can know that: (1) the proposed DR-MUSIC algorithm can give the parameter estimation effectively; (2) the performance of proposed array is better than that of PSFDA-MIMO radar, especially for the CRLB of range estimation.

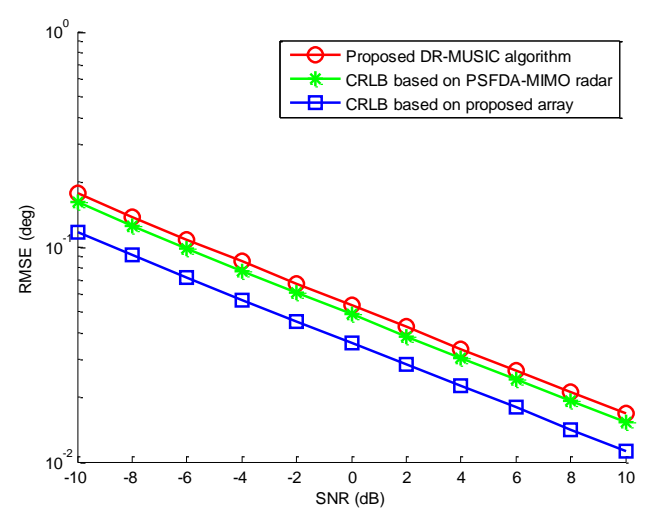

(a) RMSE of DOA estimation versus SNR.

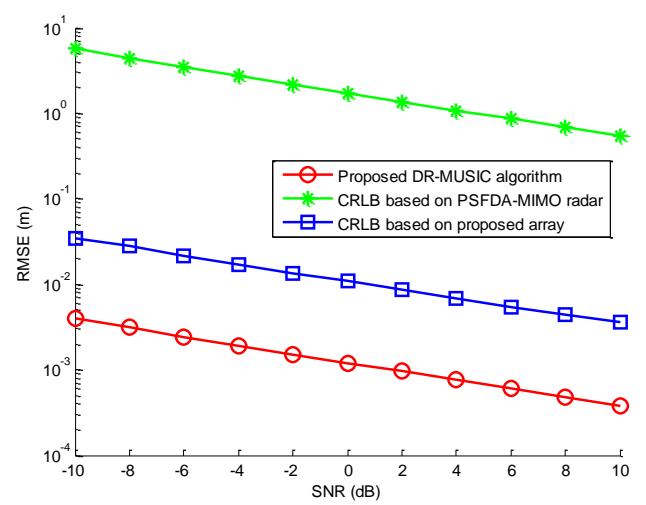

(b) RMSE of range estimation versus SNR.

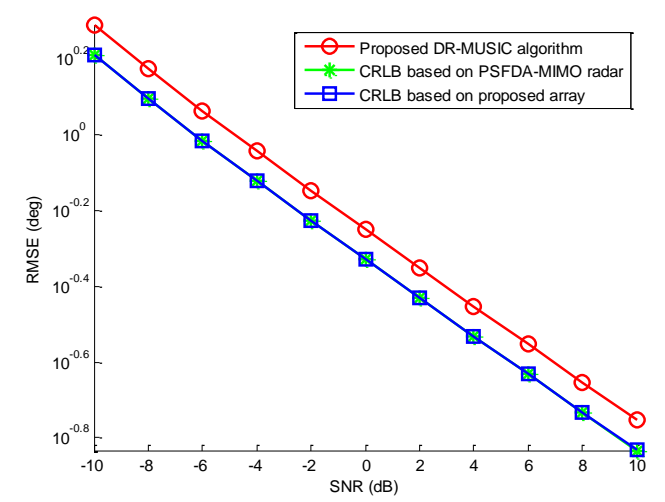

(c) RMSE of auxiliary polarization angle estimation versus SNR. 


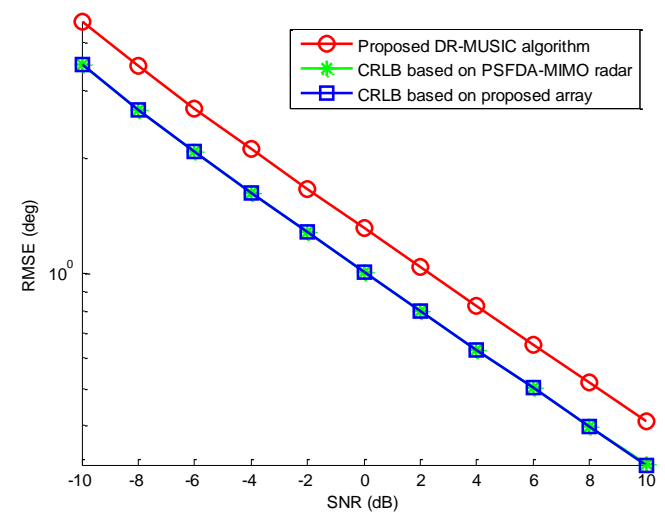

(d) RMSE of polarization phase difference estimation versus SNR.

Fig. 4. RMSE of parameter estimation versus SNR.

\section{Conclusions}

In this paper, parameter estimation with SS-PSRFDAMIMO radar has been investigated. The proposed DRMUSIC algorithm for unambiguous parameter estimation with proposed array work well. Furthermore, the CRLB of range estimation with the proposed array is much lower than that of range estimation with PSFDAMIMO radar. It is potential to improve the resolution of range.

\section{References}

1. B. Li, W. Bai, G. Zheng, X. He, Int J E (under review)

2. Y. Liu, R. Hang, L. Wang, A. Nehorai, IEEE J. Sel. Top. Signal Process., 11, 295-308 (2017)

3. K. T. Wong, M. D. Zoltowski, IEEE Trans. Antennas Propag., 45, 1467-1474 (1997)

4. J. Xu, G. Liao, S. Zhu, L. Huang, H. C. So, IEEE Trans. Signal Process., 63, 3396-3410 (2015)

5. Y. Wang, X. Mao, J. Zhang, H. Hong, Radar Conference, 0507-0511 (2014)

6. H. Chen, H. Shao, W. Wang, ICASSP, 2891-2895 (2016)

7. X. Zhang, Matrix Analysis and Applications, Beijing: Tsinghua University Press. (2004) 